\title{
Avaliação da atividade inibidora do diflubenzuron na ecdise das larvas de Aedes aegypti (Linnaeus, 1762) (Diptera, Culicidae)
}

\author{
Evaluation of the inhibiting activity of the diflubenzuron on the ecdysis of \\ larvae of Aedes aegypti (Linnaeus, 1762) (Diptera, Culicidae)
}

\author{
Flávia Martins ${ }^{1}$ e Ionizete Garcia da Silva ${ }^{2}$
}

\begin{abstract}
RESUM0
Foi avaliada a atividadeinibidora do diflubenzuron na ecdise delarvas do Aedes aegypti, visando à utilização desse produto no controle desse mosquito. Além disso, conhecer a interação do produto com o tipo de criadouro e a suscetibilidade do mosquito. Os bioensaios foram realizados em um fundo de quintal de residência, em sete tipos potenciais de criadouros artificiais: pneu, vidro, cimentoamianto, cimento, lata, plástico ecerâmica. Para cada tipo de criadouro colocaram-se 20 larvas decada estádio do Aedes aegypti. Omesmo número delarvas foi utilizado para o controle. Foram feitas nove réplicas e asleituras de mortalidade foram em intervalos de 24 horas, após o início dos experimentos, atéatingir oíndice de 100\%. Isto foi obtido a 1 ppm. Năo houve diferença significativa entre os períodos médios de sobrevivência daslarvas enem entre os diferentes tipos de criadouros. Houvediferençassignificativas entreos estádios, sendo $03^{\circ} 0$ mais tolerante. Constatou-se também que as concentrações não interagiram com os estádios etiposdecriadouros, ao nível de $5 \%$.
\end{abstract}

Palavras-chaves: Aedes aegypti. Diflubenzuron. Regulador de crescimento. Controle.

\begin{abstract}
The inhibiting activity of diflubenzuron on the ecdysis of Aedes aegypti larvae was evaluated, with a view to using this product in mosquito control. This study also aimed to determine the interaction between this product, the type of artificial containers and the susceptibility of the mosquito. Bioassays were carried out in the backyard of a residence, using seven kinds of artificial habitats: tires, glass, concrete roofing, cans, plastic containers, cement and pottery. In each kind of artificial habitat, 20 Aedes aegypti larvae in the $4^{\text {th }}$ instar were set. The same number of larvae was used as a control. Each test was repeated five times and the observation of mortality was done once every 24 hours, until $100 \%$ mortality was reached at $1 \mathrm{ppm}$. There was no significant difference between the main surveillance periods of the larvae, nor between the various kinds of artificial habitats. A significant difference was found between the instars, in that the $3^{\text {rd }}$ instar was the most resistant to diflubenzuron inhibiting activity. It was also shown that concentrations did not interact with instars or material of the artificial habitats at the $5 \%$ significance level.
\end{abstract}

Key-words: Aedes aegypti. Diflubenzuron. Insect growth regulator. Control.

Os reguladores de crescimento de insetos formam um grupo de inseticidas de nova geração que causam modificações fisiológicas e morfológicas, durante o desenvolvimento do

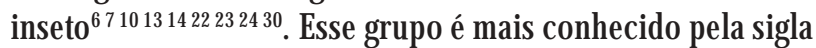
IGR (insect growth regulator). Os IGRs surgiram na década de 70 como um novo grupo de inseticidas de ação mais específica e menor toxicidade para mamíferos do que os outrosinseticidas ${ }^{23} 30$, pois atuam seletivamente ao interromper o desenvolvimento e 0 crescimento ao invés de intoxicação direta.

0 diflubenzuron é um IGR que age pela inibição da síntese de quitina durante 0 estágio imaturo do inseto, tem sido comercializado em muito países com o nome dimilin ${ }^{101617182730}$. Esse produto é

1. Seção de Entomologia da Secretaria da Saúde do Estado de Goiás. 2. Laboratório de Biologia e Fisiologia de Insetos e Xenodiagnóstico do Instituto de Patologia Tropical e Saúde Pública (IPTSP) da UFG, Goiânia, GO.

Apoio Financeiro: FUNAPE.

Endereço para correspondência: Dr. Ionizete Garcia da Silva. Departamento Microbiologia, Imunologia, Parasitologia e Patologia/IPTSP/UFG. Caixa Postal 131, 74001-970 Goiânia, G0.

Tel: 5562 209-6128; Fax:55 62 261-2077.

e-mail:ionizete@iptsp.ufg.br

Recebido para publicação em 14/3/2003

Aceito em 20/12/2003 
utilizado na agricultura desde a década de 90, contra insetos pragas da agricultura das ordens: Coleopter ${ }^{21}$, Lepidoptera ${ }^{33} \mathrm{e}$ Ortoptera ${ }^{3}$.

Para osinsetos deinteresse na área da saúde existem vários estudos, mostrando os efeitos inseticidas do diflubenzuron, com a possibilidade de uso no controle ${ }^{1246781527}$. Neste estudo, 0 alvo foi o Aedesaegypti, mosquito com comportamento predominantemente antropofilico e urbano em toda região cosmotropical.

0 Aedes aegypti teve papel importante na re-emergência nas infecções causadas pelos vírus do dengue em suas diversas formas clínicas. Essa virose tornou-se um dos mais graves problemas de saúde pública no mundo. Cerca de 3,5 bilhões de pessoas vivem expostas a esse mosquito ${ }^{11} 1920253132$ na faixa cosmotropical, constituindo um grande desafio para os serviços de saúde, devido a inexistência, até o momento, de uma vacina eficaz para 0 uso preventivo contra 0 dengue ${ }^{1234}$.

Oúnico meio disponivel na prevenção de dengue é o combate ao vetor, o qual está limitado na eliminação dos seus criadouros e ao combate químico. Este último tem induzido ao desenvolvimento de populações resistentes ${ }^{5} 152628$, sendo assim necessário pesquisas de produtos novos e mais seguros ao meio ambiente, como alternativa estratégica de controle vetorial. Dentre essas 0 diflubenzuron poderá constituir-se numa importante ferramenta, através de sua atividade inibidora na ecdise das larvas. Esse produto é praticamente atóxico para mamíferos, biodegradável, não sistêmico e de baixo impacto ambiental ${ }^{6}$.

\section{MATERIAL E MÉTODOS}

Criaram-se larvas de A aegypti de acordo com metodologiajá definida ${ }^{29}$, numa câmara biológica climatizada a $28^{\circ} \pm 1^{\circ} \mathrm{C}$, com umidade relativa de $80 \pm 5 \%$ e fotofase aproximada de 12 horas. Em outra câmara, climatizada similarmente, iniciaram-se os testes de suscetibilidade do A aegypti ao diflubenzuron 25\% (Champion Farmoquímica Ltda, Brasil). Pesou-se 0,25 g do produto em balança analítica de precisão e, em seguida, dissolvido em 100 $\mu$ lde água destilada com 0 auxílio de um agitador magnético durante 30 minutos, obtendo-se uma solução estoque. Desta solução utilizaram-se 200, 160, 80, 40 e 20 $\mu$ l, que foram diluídos em 500ml de água destilada, obtendo-se respectivamente as concentrações de: 1, 0,8 , 0,4 , 0,2 e 0,1 ppm utilizadas nos bioensaios.

Usaram-se 20 larvas para cada estádio e concentração, com 9 réplicas, em copos plásticos descartáveis, com 30ml de solução em cada um. Com auxílio de uma pipeta plástica fazia-se a coleta das larvas da bacia de criação para esses copos. No grupo controle, as larvas foram colocadas na mesma quantidade e no mesmo volume de água destilada.

As leituras de mortalidade foram realizadas em intervalos de 24 horas após o início dos experimentos, até atingir o índice de 100\%. Nas observações diárias as larvas mortas foram retiradas dos copos com 0 auxílio de uma pipeta plástica e foram consideradas mortas quando havia ausência total de movimentos, com escurecimento do corpo e cápsula cefálica.

Os experimentos de campo foram realizados em fundo de quintal, em sete tipos de recipientes, com o mesmo no-de larvas e repetições do laboratório. Os criadouros potenciais utilizados foram: cimento-amianto, cimento, cerâmica, vidro, lata, pneu e plástico, com o objetivo de verificar a interferência do tipo de recipiente e as variáveis climáticas com 0 produto. Em cada criadouro colocaram-se 2 litros de solução, preparadas com água da rede pública de abastecimento. As leituras de mortalidade foram feitas obedecendo os mesmos critérios do laboratório.

Para a análise de sobrevivência das larvas usou-se o método não paramétrico de Kaplan Meier, e para comparação do período inibitório da ecdise, nos quatro estádios larvais do A. aegypti, utilizou-se a análise de variância seguida do teste de Tukey, usando o programa Statistical Analisys Sistem ( SAS) .

\section{RESULTAD0S}

0 diflubenzuron apresentou atividade larvicida em todos os estádios, tanto nos bioensaios de laboratório quanto nos de campo. Os períodos médios de tempo para que o diflubenzuron alcançasse a eficácia de 100\% das larvas de A. aegypti, são apresentados nas Tabelas 1 e 2 .

Tabela 1 - Período médio (em dias) de sobrevivência das larvas de Aedes aegypti, à ação inibidora do diflubenzuron na ecdise, com mortalidade de 100\%, em laboratório.

\begin{tabular}{lccrr}
\hline $\begin{array}{l}\text { Concentrações } \\
(\mathrm{ppm})\end{array}$ & $1^{\circ}$ estádio & $2^{\circ}$ estádio & $3^{\circ}$ estádio & $4^{\circ}$ estádio \\
\hline 0,1 & $6,1 \pm 0,57 \mathrm{a}$ & $5,1 \pm 0,65 \mathrm{a}$ & $8,0 \pm 1,93 \mathrm{~b}$ & $8,1 \pm 0,82 \mathrm{~b}$ \\
0,2 & $5,9 \pm 0,54 \mathrm{a}$ & $5,3 \pm 0,33 \mathrm{a}$ & $8,4 \pm 2,01 \mathrm{~b}$ & $8,0 \pm 0,79 \mathrm{c}$ \\
04 & $5,6 \pm 0,43 \mathrm{a}$ & $5,1 \pm 0,31 \mathrm{a}$ & $7,4 \pm 1,77 \mathrm{~b}$ & $7,5 \pm 1,34 \mathrm{~b}$ \\
0,8 & $6,1 \pm 0,53 \mathrm{a}$ & $5,6 \pm 0,44 \mathrm{a}$ & $8,1 \pm 1,62 \mathrm{~b}$ & $7,3 \pm 0,73 \mathrm{c}$ \\
1,0 & $5,1 \pm 0,47 \mathrm{a}$ & $5,3 \pm 0,69 \mathrm{a}$ & $7,4 \pm 1,50 \mathrm{~b}$ & $7,0 \pm 0,50 \mathrm{c}$ \\
\hline
\end{tabular}

Obs: As médias seguidas da mesma letra, não apresentam diferença significativa entre si, pela análise de variância e 0 teste de Tukey, ao nível de 5\%. \pm erropadrão da média.

Tabela 2 - Período médio (em dias) de sobrevivência das larvas de Aedes aegypti, à ação inibidora do diflubenzuron na ecdise, com mortalidade de $100 \%$ até $03{ }^{\circ}$ estádio, em bioensaios de campo.

\begin{tabular}{lrrrrr}
\hline \multicolumn{2}{l}{ Concentrações } & & & mortalidade \\
$(\mathrm{ppm})$ & $1^{\circ}$ estádio & $2^{\circ}$ estádio & $3^{\circ}$ estádio & $4^{\circ}$ estádio & $\%$ \\
\hline 0,1 & $8,6 \pm 0,35 \mathrm{a}$ & $7,6 \pm 0,35 \mathrm{~b}$ & $10,4 \pm 0,22 \mathrm{c}$ & $10,2 \pm 0,20 \mathrm{c}$ & 98,0 \\
0,2 & $8,4 \pm 0,22 \mathrm{a}$ & $7,8 \pm 0,33 \mathrm{~b}$ & $10,8 \pm 0,52 \mathrm{c}$ & $11,2 \pm 0,18 \mathrm{c}$ & 98,0 \\
0,4 & $8,2 \pm 0,18 \mathrm{a}$ & $7,8 \pm 2,23 \mathrm{a}$ & $9,0 \pm 0,40 \mathrm{a}$ & $10,2 \pm 0,43 \mathrm{c}$ & 98,0 \\
0,8 & $8,8 \pm 0,18 \mathrm{a}$ & $8,2 \pm 0,33 \mathrm{~b}$ & $10,8 \pm 1,07 \mathrm{c}$ & $9,6 \pm 0,21 \mathrm{c}$ & 99,0 \\
1,0 & $8,2 \pm 0,33 \mathrm{a}$ & $8,4 \pm 0,35 \mathrm{a}$ & $10,8 \pm 0,97 \mathrm{c}$ & $10,4 \pm 1,01 \mathrm{c}$ & 100,0
\end{tabular}

Obs: As médias seguidas da mesma letra, não apresentam diferença significativa entre si, pela análise de variância e 0 teste de Tukey, ao nível de 5\%. \pm erropadrão da média

As leituras de sobrevivência das larvas do A aegypti foram avaliadas em intervalos de 24 horas após o início dos experimentos até atingirem 0 índice de 100\% de mortalidade. Esta ocorria pela interferência do diflubenzuron na síntese e/ou reabsorção da quitina, no momento da muda, não sendo uma intoxicação direta nas larvas, como ocorrem com os inseticidas químicos dos grupos clorados, fosforados, carbamatos e piretróides.

No laboratório, a mortalidade de $100 \%$ das larvas de A aegypti foi obtida em todas as concentrações estudadas, de 0,$1 ; 0,2 ; 0,4 ; 0,8$ 
e 1 ppm. Não houve diferença significativa entre os períodos médios de tempo de sobrevivência das larvas nessas concentrações. Houve diferenças significativas entre os estádios, sendo $03^{\circ} 0$ mais tolerante à ação inibidora do diflubenzuron.

Nos bioensaios de campo, foi constatado que as concentrações não interagiram com os tipos de criadouros $(p<0,05)$. Constatou também, que a inibição da síntese e/ou reabsorção total da quitina ocorria na concentração de 1 ppm, com 100\% de mortalidade. Nas outras concentrações houve sobrevivência, apenas no $4^{\circ}$ estádio, entre 1 e $2 \%$, provavelmente isso ocorreu pela interferência de variáveis como a luz, temperatura, umidade e hormônios que aparecem na formação da pupa. A temperatura média foi de $24 \pm 7,2^{\circ} \mathrm{C}$ e a umidade relativa do ar foi de $54 \pm 7,9 \%$. Dessa forma, estes resultados evidenciam a concentração de $1 \mathrm{ppm}$, como dose referência para mortalidade total, dando perspectivas para utilização desse IGR no combate do A aegypti.

\section{DISCUSSÃ0}

Os resultados podem ser considerados como concordantes com os obtidos em Taiwan ${ }^{2}$, China, pela similaridade da dose e da mortalidade, por ser estável nessa concentração, à luz, temperatura e ao $\mathrm{pH}$ da água.

Os resultados obtidos neste trabalho nas concentrações de 0,1 ppm, mostraram redução da emergência entre 85 a 90\%, enquanto a avaliação desse mesmo produto na Índia, para Culex quinquefasciatus mostrou, nessa dosagem, uma redução de $48 \%$. Mas quando se utilizaram doses mais altas, de 0,2 e 0,4 ppm, os índices de mortalidade variaram entre 80 e $96 \%$, dependendo da espécie de mosquito estudada, embora nesse estudo não tenha sido incluída a espécie A aegypti. Resultados semelhantes foram obtidos na Califórnia ${ }^{27}$ (USA) para Psorophora confinis, pelas doses de 0,11 e 0,25 ppm.

Neste trabalho, 0 diflubenzuron foi dissolvido em água para todos os bioensaios. As concentrações utilizadas foram maiores do que as de outros autores ${ }^{67810}$, provavelmente pelo fato de ter sido solubilizado o mesmo produto em acetona e testadas apenas em laboratório. Além disso, havia emergência de adultos com resultados nada animadores com relação a fecundidade e fertilidade, que variava com as concentrações utilizadas e não afetando a geração tratada. Havia diminuição da fecundidade e da fertilidade na geração subseqüente ${ }^{7813}$. Mas as experiências de Taiwan ${ }^{5}$ no combate ao A aegypti, nas doses de 1-5 ppm com o diflubenzuron, corroboram os resultados sugeridos neste trabalho.

Para 0 uso do diflubenzuron em futuro próximo, ressaltase a necessidade de treinamento dos agentes de saúde e a conscientização da comunidade, pelo fato do produto não ser veneno e a morte da larva ocorrer pela inibição da ecdise. Essa morte será mais lenta do que os inseticidas considerados convencionais, sendo cerca de uma semana. E isso, provavelmente, induzirá uma desconfiança à população, com relação a eficácia do produto, dificultando o trabalho de combate ao mosquito. Mas, o uso imediato desse produto seria recomendado naquelas localidades onde A. aegypti apresenta resistência ao temephos, que é o larvicida utilizado ostensivamente na faixa cosmotropical.
Outro fator estimulante para uso desse produto é o fato de ser biodegradável e não cumulativo no organismo, por não ser assimilado pelo intestino de vertebrados ${ }^{22}$. 0 fato negativo seria a sua ação em mudas de outros insetos que compartilham criadouros naturais. Com relação ao A. aegypti esse aspecto é irrelevante, pois esse mosquito apresenta hábitos urbanos, desenvolvendo-se fundamentalmente em criadouros artificiais, oriundos de embalagens e artefatos descartáveis e que raramente compartilham esses criadouros com outros insetos. Com relação à água de consumo humano, proveniente de poços freáticos e armazenada em caixas d'água e similares, 0 uso não oferece perigo, pois o diflubenzuron atua apenas na síntese de quitina, durante o processo de muda e essa é uma substância restrita aos artrópodes.

\section{REFERÊNCIAS BIBLIOGRÁFICAS}

1. Chamberlain WF. Insect growth regulating agents for control of arthropods of medical and veterinary importance. Journal Medical Entomology 12:395400, 1975.

2. Chau-Mei HO, Shy-Huey WU, Chin-Chen WU. Evaluation of the control of mosquitoes with insect growth regulators. Kaohsiung Journal Medical Science 6:366-374, 1990.

3. Delgado FX, Britton JH, Ansager JA, Swearingen W. Field assessment of Beauveria bassiana (Balsamo) vuillemin and potential synergism with diflubenzuron for control of savanna grasshopper complex (Orthoptera) in Mali. Journal of Invertebrate Pathology 73:34-39, 1999.

4. Dorta AM, Chiong RT, Ortega AN, Quinones FAG. Estudio de la sensibilidade al Dimilin (Diflubenzuron) en una cepa de Aedes aegypti Linnaeus y de Culex quinquefasciatus Say criadas en el laboratorio. Revista Cubana de Medicina Tropical 41:56-63, 1989.

5. Failloux $A B$, Ung $A R$, Pasteur N. Insecticide susceptibility in mosquitoes (Diptera: Culicidae) from French Polynesia. Journal Medical Entomology 31:639-644, 1994.

6. Fournet F, Sannier C, Monteny N. Effect of the insect growth regulators OMS 2017 and Diflubenzuron on the reproductive potential of Aedes aegypti. Journal of the American Mosquito Control Association 9:426-430, 1993.

7. Fournet F, Sannier C, Monteny N. Effeccts of two insect growth regulators on the susceptibility of Aedes aegypti (Diptera: Culicidae) to Molinema dessetae (Nematoda: Filarioidea). Journal of the American Mosquito Control Association 13: 40-42, 1997

8. Fournet F, Sannier C, Moriniere M, Porcheron P, Monteny N. Effects of two insect growth regulators on ecdysteroid production in Aedes aegypti (Diptera: Culicidae). Journal Medical Entomology 32:58-593, 1995.

9. Franco 0. História da febre amarela no Brasil. Impressora Brasileira Ltda, Rio de Janeiro, 1969.

10. Graf JF. The role of insect growth regulators in arthropod control. Parasitology Today 9: 471-474, 1993.

11. Guzman MGT, Kouri GF, Bravo JRG. Emergence of dengue hemorrhagic fever in the Americas. Reemergence of dengue. Revista Cubana de Medicina Tropical 51:5-13, 1999.

12. Kitamura T. A rapporteurs summary: research on dengue vaccine. Tropical Medicine 35:325-330, 1993.

13. Kunz SE, Harris RL, Hogan BF, Wright JE. Inhibition of development in a population of horn flies treated with diflubenzuron. Journal of Economic Entomology 70:298-301, 1977.

14. Lyra JRM, Ferraz JMG, Silva APP. Acción de inhibidores de la síntesis de la quitina en la reprodución de Spodoptera littoralis (Boisd.) (Lepidoptera: Noctuidae). Anais da Sociedade Entomológica do Brasil 27:569-576, 1998.

15. Macoris MLG, Camargo MF, Silva IG, Takaku L, Andrighetti MT. Modificação da suscetibilidade de Aedes ( Stegomyia) aegypti ao temephos. Revista de Patologia Tropical 24:31-40, 1995. 
16. Martin PJS, Clark JM, Edman JD. Preliminary study of synergism of acid rain and diflubenzuron. Bulletin of Environmental Contamination and Toxicology 54:833-836, 1995.

17. Martínez AF. Inhibidores de síntesis de quitina: Control de problaciones de mosquitos con productos biorracionales. Revista da Sociedade de Entomologia Argentina 58:232-237, 1999.

18. Mekuria Y, Gwinn TA, Williams DC, Tidwell MA. Insecticide susceptibility of Aedes aegypti from Santo Domingo Dominican Republican. Journal of the American Mosquito Control Association 79:69-72, 1991.

19. Ministério da Saúde. Boletim Eletrônico Epidemiológico. http:// www.funasa.gov.br, 2001.

20. Ministério da Saúde. Boletim Eletrônico Epidemiológico. http:// www.funasa.gov.br, 2002.

21. Mscasland CS, Cooper RJ, Barnum DA. Implications for the use diflubenzuron to reduce arthropod populations inhabiting evaporation ponds of the San Joaquin Valley, California. Bulletin of Environmental Contamination and Toxicology 60:702-708, 1998.

22. Mulla MS. The future of insect growth regulators in vectors control. Journal of the American Mosquito Control Association 11:269-273, 1995.

23. Mulla MS, Darwazeh HA. New insect growth regulators against flood and stagnant water mosquitoes-effects on non target organisms. Mosquitoes News 39:746-755, 1979.

24. Mulla MS, Darwazeh HA, Schreiber ET. Impact of new insect growth regulators and their formulations on mosquito larval development in impoundment and floodwater habitats. Journal of the American Mosquito Control Association 5:15-20, 1989.
25. Pinheiro FP, Corber SJ. Global situation of dengue and dengue haemorrhagic fever and its emergence in the Americas. World Heath Statq 50:161-169, 1997.

26. Rawlins SC. Spatial distribution of insecticide resistance in Caribbean populations of Aedes aegypti and its significance. Revista Panamericana Salud Publica 4:243-251, 1998.

27. Schaefer CH. A practical evaluation of TH-6040 ( Dimilin) as a mosquito control agent in California. Journal of Economic Entomology 28:183-185, 1975.

28. Schaefer CH, Miura T, Wilder WH, Mulligan FS. New substituted benzamines with promising activity against mosquitoes. Journal of Economic Entomology 71:427-430, 1978.

29. Silva HHG, Silva IG, Lira KS. Metodologia de criação, manutenção de adultos e estocagem de ovos de Aedes aegypti (Linnaeus, 1972) em laboratório. Revista de Patologia Tropical 27:51-63, 1998.

30. Silva JJ, Mendes J. Effect of diflubenzuron on stages of Hematobia irritans (L.) (Diptera, Muscidae) in Uberlândia, State of Minas Gerais, Brasil. Memórias do Instituto Oswaldo Cruz 97:679-682, 2002.

31. Tauil PL. Urbanização e ecologia do dengue. Cadernos de Saúde Pública 17:99-102, 2001.

32. Tauil PL. Aspectos críticos do controle do dengue no Brasil. Cadernos de Saúde Pública 18:867-871, 2002.

33. Villanueva-Jimenez JÁ, Hoy MA, Davies FS. Field evaluation of integrated pest management-compatible pesticides for the citrus leafminer Phyllocnistis citrella (Lepidoptera: Gracillariidae) and its parasitoid Ageniaspis citricola (Hymenoptera: Encyrtidae). Journal of Economic Entomology 93:357-367, 2000.

34. Yasui K. Strategies of dengue vaccine development by WHO using new biotechnology. Tropical Medicine 35:233-241, 1993. 